\title{
Elimination of Frequency Modulated Magnetic Field Suppression of Melanoma Cell Proliferation by Simultaneous Exposure to a Pattern Associated With Memory in Mammals
}

\author{
Nirosha J. Murugan, Lukasz M. Karbowski and Michael A. Persinger \\ Bioquantum Biomolecular Laboratory, Biomolecular Sciences Program, Laurentian University, Sudbury, Ontario, P3E 2C6 Canada
}

Corresponding author: Michael A. Persinger, Bioquantum Biomolecular Laboratory, Biomolecular Sciences Program, Laurentian University, Sudbury, Ontario, P3E 2C6 Canada, Tel: 01-705-675-4824; Fax: 01-705-671-3844; E-mail: mpersinger@laurentian.ca

Received: 08 June 2016; Accepted: 14 June 2016; Published: 20 June 2016

Citation: Murugan NJ, Karbowski LM, Persinger MA. Elimination of Frequency Modulated Magnetic Field Suppression of Melanoma Cell Proliferation by Simultaneous Exposure to a Pattern Associated With Memory in Mammals. Arch Can Res. 2016, 4: 2.

\section{Abstract}

Strategic whole body application of physiologicallypatterned, weak magnetic fields has been evolving as a third option to treat cancer. Unlike chemical therapies and ionizing radiation physiologically-patterned magnetic fields diminish the growth of only malignant cells but not normal cells. In addition these fields provide some analgesic relief. To discern if competing magnetic fields might abolish the beneficial effects mouse melanoma cells in culture were exposed for 1 hour per day for 5 days to decelerating frequency modulated patterned magnetic fields that are well known for their growth suppressive effects mediated by T-Type calcium channels. Typical suppression rates were obtained. Exposure to a second pattern that evokes LTP (long term potentiation) in hippocampal slices and is the primary physical correlate of memory, produced minimal effects. However when the effective daily frequency-modulated hourly exposures were preceded by only 30 min of LTP patterned magnetic fields the growth suppression effects were completely abolished. These results indicate certain patterned fields cancel the beneficial effects and that the processes that underlie proliferation of malignant cells and memory consolidation in nervous tissue may share characteristics.

Keywords: Melanoma cells; Weak magnetic field effects; Frequency-modulated pulse; Thomas pattern; LTP (long term potentiaton); Synergistic cancellation

\section{Introduction}

Diminishment or (optimally) elimination of malignant cell growth and proliferation is the primary goal of oncological intervention. Until recently the two primary methods for diminishing malignant cell proliferation have been chemical therapies and ionizing radiation. Unfortunately large numbers of normal or non-malignant cells are disrupted or destroyed by these methods as well. In addition malignant cells appear to habituate or to become resistant to these stimuli. A third method has been developed that employs physiologicallypatterned magnetic fields. It diminishes the growth of only cancer cells without adversely affecting normal cells. Several experimenters [1-4], employing different equipment and magnetic field patterns, have demonstrated reliable and consistent reduction ( $50 \%)$ in growth within malignant cell cultures. If this technology is to be employed as a substitute for traditional methods then the variables that interfere with its beneficial effects should be isolated and described before routine treatment begins. Here we show that a specific type of magnetic field pattern known to simulate long-term potentiation in brain tissue [5-6] and is considered the fundamental substrate for learning and memory eliminated the beneficial effects of an established anticancer magnetic field pattern [1].

On the basis of theoretical considerations and quantitative solutions there is sound evidence that some temporally complex magnetic fields within the range of 1 to $5 \mu \mathrm{T}$ should specifically affect malignant cells. Persinger and Lafrenie [7] have argued that the conspicuous near-Nernst level resting potentials of the plasma membranes of cancer cells, compared to the greater polarity of normal or non-malignant cells, increases their vulnerability to factors coupled to energies associated with entropy, information, and cosmic microwave background sources [8]. In a series of remarkably innovative experiments Buckner and Lafrenie [9] found that magnetic fields generated from photocoupler, digital to analogue computerized sequences [10] diminished the growth rate in cell cultures of approximately a dozen different types of human and animal cancer cells but did not affect normal cells. The consistent diminishment from one hour exposures per day over five days was about $35 \%$ to $50 \%$ relative to sham-field exposed cancer cells. There was no diminishment in normal cells.

Unlike most approaches that employed function generators Buckner and Lafrenie [9] applied the technology developed by Koren et al. [10] that utilized the transformation of numbers from 0 through 256 to equivalent voltages of -5 to $+5 \mathrm{~V}$ from specific optocoupler devices that operated the pairs of solenoids to which the cells were exposed. As was noted for the marked analgesic effects of these fields that simulated the effects of therapeutic dosages of morphine in rats [11] the efficacy of the malignant cell suppression effects was dependent upon the point duration. This is the duration in milliseconds that each value between 0 and 256 was 
converted to voltages applied to the solenoids to produce the field. The optimal point duration has been $3 \mathrm{~ms}$. Point durations that are less or more than this value have not been effective. Consistent with the Persinger and Lafrenie [7] hypothesis there is empirical evidence that this $3 \mathrm{~ms}$ point duration may reflect a fundamental property of proton function within the structure of space $[12,13]$.

The specific pattern of magnetic field that has been most frequently employed successfully is a decelerating frequency modulated configuration popularly labeled the Thomas pattern after Dr. Alex Thomas who was one of the students in the laboratory at time of its construction. It is shown in Figure 1. Applications of this pattern with intensities around $3 \mu \mathrm{T}$ have been shown to significantly influence the influx of calcium ions into melanoma cells. Buckner et al. [14] verified that the cellular mechanism for this effect was strongly dependent upon the operation of T-Type calcium channels. However other calculations have suggested that protons or proton channels coupled to these channels may be significant modulators. T-type channels are also strongly correlated with enhanced spontaneous electrical activity that is associated with epilepsy [15] in more extreme forms and memory-related consolidation within normal frameworks [16]. LTP or long-term potentiation is the label for a class of electrophysiological phenomena found primarily in neurons or paraneurons that has been considered the primary substrate for the representation of experience, i.e., memory. It is primarily reflected as the maintained diminished threshold in scattered spatial aggregates of cells to respond to stimulus configurations that have been encouraged previously.

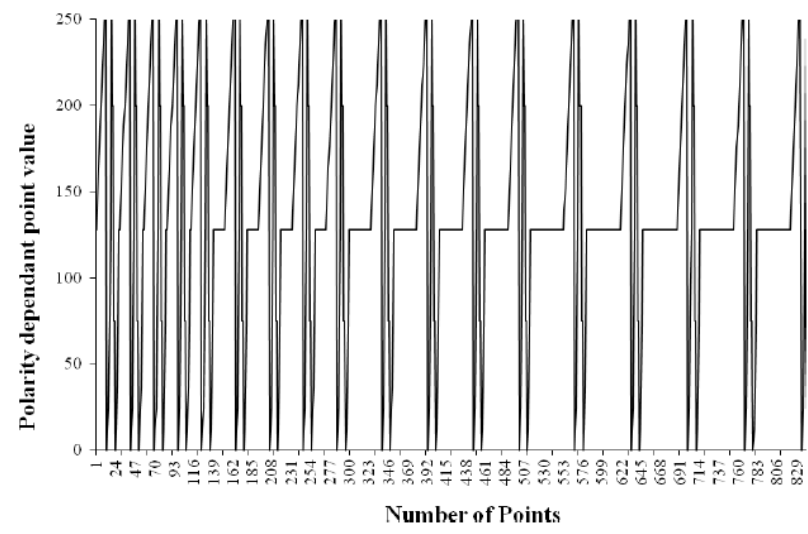

Figure 1 The temporal structure of the decelerating frequency-modulated magnetic field (Thomas pulse) pattern composed of serial points (between 0 and 256) each with a duration of $3 \mathrm{~ms}$. Vertical axis reflects the values between 0 and 256 while the horizontal axis is the number of points.

A review of the scientific literature in oncology and in learning reveals the striking similarity of the common biomolecular signaling pathways involved with memory consolidation and proliferation of malignant cells [17]. It may not be serendipitous that LTP occurs primarily within the relatively unique neuron-type cell which does not divide following differentiation. If the conditions that contribute to cancer proliferation and memory consolidation in mammals were too similar then any process that enhanced LTP activity within cerebral function could encourage malignancy. We digitized the same pattern that when employed as electrical stimulation produced LTP in hippocampal slices [5]. St-Pierre and Persinger [18] reported results that may support this supposition. They found that rats exposed prenatally to a very complex magnetic field (the lindagene pattern) composed of 10,000 points with more than a dozen distinct patterns (one of which was the Thomas pattern) where each pattern was punctuated by $200 \mathrm{~ms}$ of a LTP sequence displayed mild organizational anomalies within the hippocampus. Some rats displayed conspicuous malformations that were similar to ontogenetic malignant growths within large areas of the hippocampus (Figure 2).

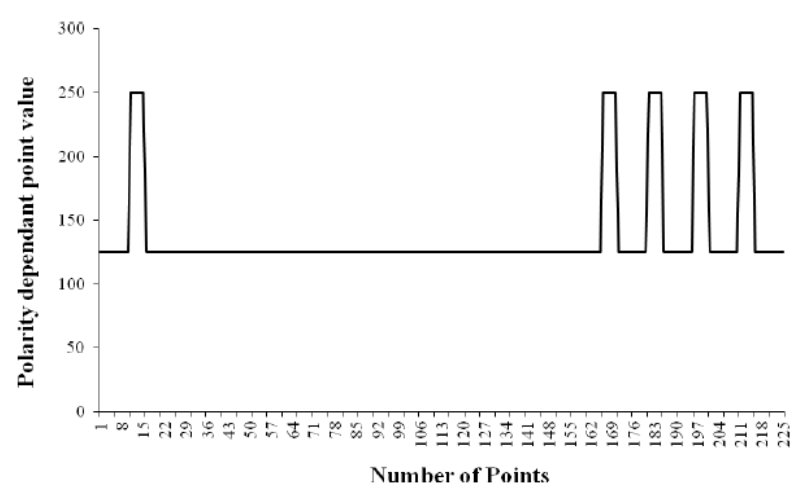

Figure 2 The LTP (Long-term potentiation) pattern which is the magnetic field equivalent of the electrical current pattern known to induce LTP in hippocampal slices. Note this pattern is only one $(+)$ polarity.

That whole body experimentally applied LTP-patterned magnetic fields could block natural brain-related activity that is associated with learning and memory was clearly demonstrated by Mach and Persinger [19]. They found that rats trained in the Morris water maze every hour and either exposed to the LTP pattern for either $30 \mathrm{~min}$ before or $30 \mathrm{~min}$ after the maze displayed markedly asymmetric deficits that were comparable to complete depolarization of the hippocampus with DC currents. Those exposed to the LTP pattern before the next training exhibited marked learning deficits. Those exposed to the same LTP pattern for $30 \mathrm{~min}$ but after the previous training did not differ appreciably from nonexposed rats. This temporal anisotropy supported the robust nature of the effect and the importance of the ongoing processes at the time of the field exposure. We reasoned that the potency of the LTP pattern might be sufficient to interact with the effects of the Thomas pattern when applied to malignant cell cultures.

\section{Methods}

All experiments were completed in triplicate. Mouse melanoma (B16 - BL6) cells were cultured onto $60 \mathrm{~mm} \times 15$ $\mathrm{mm}$ dishes in DMEM (Dulbecco's Modified Eagle Medium) that 
contained $10 \%$ fetal bovine albumin and antibiotics in a $5 \%$ carbon dioxide environment at $37^{\circ} \mathrm{C}$ in standard incubators. Cells were exposed to one of four conditions. They were: 1) sham field, 2) Thomas pattern (3 ms point duration), 3) LTP (3 ms point duration), or, 4) LTP+Thomas (each $3 \mathrm{~ms}$ point durations). Cells were exposed for 1 hour per day for 5 consecutive days, with the exception of condition 4. Here a 30 min LTP exposure preceded the $1 \mathrm{hr}$ Thomas pattern exposure. After five days the cells were harvested and counted by standard hemocytometer quantification methods using Trypan blue staining. For each sampled plate eight separate counts were obtained and averaged. The procedure was similar to our hundreds of previous experiments [20].

In summary 0.5 to 1 million mouse melanoma B16 cells were exposed in stacks of six plates within custom-constructed acrylic exposure boxes which were $10 \mathrm{~cm} \times 10 \mathrm{~cm} \times 10 \mathrm{~cm}$. On each side of the box a Radioshack Reed Relay (SPST-5VDC; 275-0232) was attached (rated 0.5 A; 125 AC; coil resistance 250 ohms, nominal current $20 \mathrm{~mA}$ ). They were encased in a plastic film canister (Figure 3). The magnetic field time-varying patterns were generated simultaneously between each of the two opposing solenoids. The strength of the computergenerated time-varying magnetic field within which the stacks of 6 plates were placed averaged $28 \mathrm{mG}$ and was based upon theoretical calculations [7].

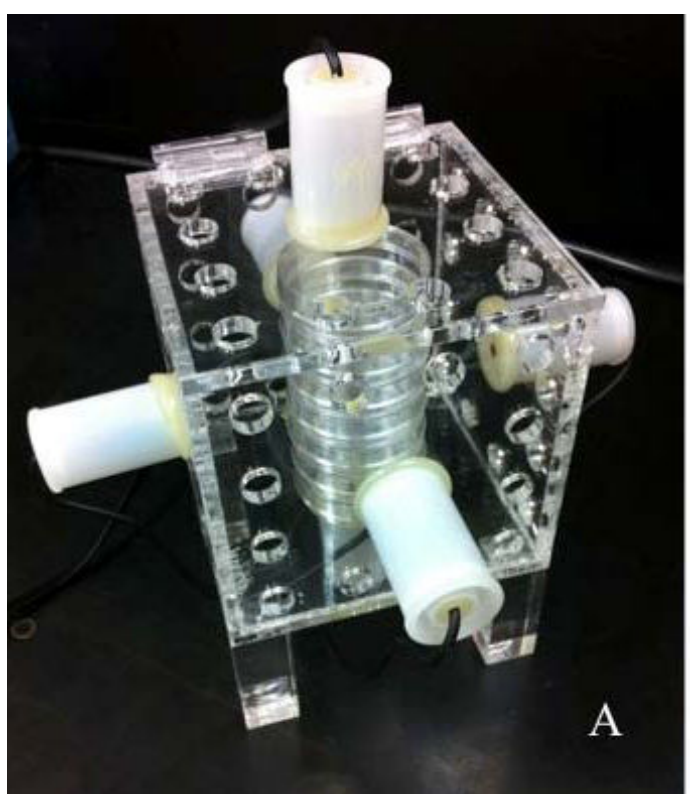

Figure 3 Example of the plastic exposure chambers for cell cultures. Each white canister contained a solenoid. Pairs of solenoids in the same plane were connected such that the field was generated in the same plane in all three planes by the input from the unique circuit composed of optocouplers, tyristors, Triac switches and LED whose temporal patterns were controlled by the software with programmable options for the point durations producing the patterns.

\section{Results}

The results were clear and consistent. As shown in Figure 4, the melanoma cells that had been exposed for 1 hour per day for five consecutive days to the frequency-modulated (Thomas) pattern displayed about a $40 \%$ reduction in numbers. This was comparable to the results reported by other experimenters. In comparison the cells exposed to the LTP pattern displayed a statistically significant (no overlap in standard errors of the means) although much weaker suppression effect compared to the sham-field controls $(p=0.004)$. However, by far the most important result was the complete elimination of the beneficial suppression effects of the Thomas pattern if the cells had been exposed to LTP pattern for only $30 \mathrm{~min}$ per day just before the $60 \mathrm{~min}$ exposure to the Thomas pattern.

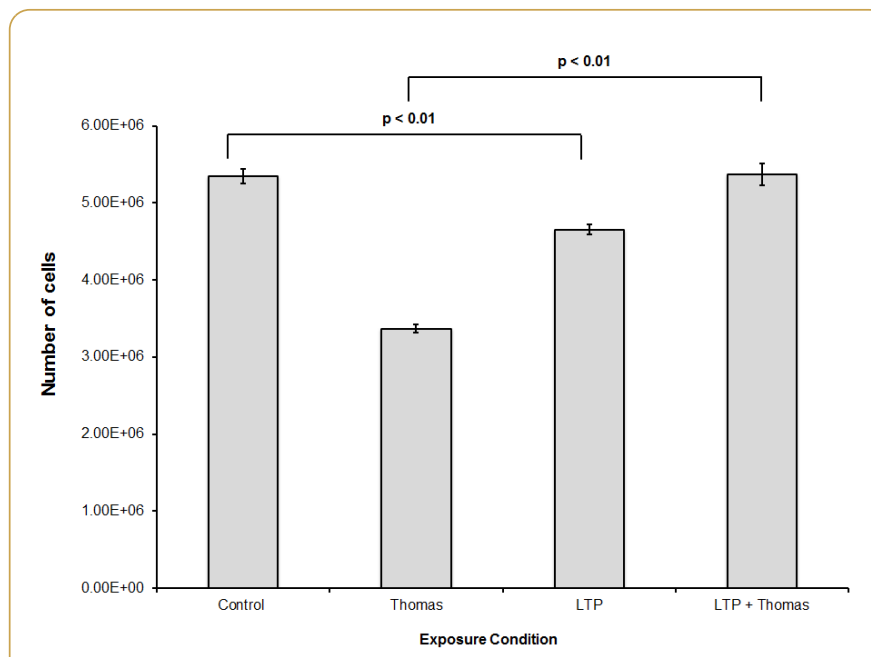

Figure 4 Numbers of cells counted after 5 days ( $1 \mathrm{hr}$ per day) exposures to either sham fields (control) or to one of three magnetic field configurations: the Thomas pattern only, the LTP pattern only, or both fields (the LTP followed by the Thomas pattern). Vertical bars indicate standard errors of the mean.

\section{Discussion}

Many other experimenters [1-4] as well as our research group have shown that exposures of malignant cells for one or more hours per day for five or so consecutively days results in consistent suppression of growth rates. Normal cells have not been affected. The optimal point duration, that is the specific time each number that generates a particular voltage and a specific strength of magnet field occurs, is $3 \mathrm{~ms}$. This point duration has been associated with the properties of protons within space [12], the duration and concentrations of the proton movements within the hydronium ion and their dynamics within proton channels [21].

The temporal specificity of the Thomas pattern that produces the suppression of malignant cell growth is very clear. Reversal of the pattern does not produce malignant cell suppression [9]. This indicates that the temporal arrow of the 
field is essential for the effect and that the potency is not nonspecific. The point duration of the successive computergenerated voltages that produce the pattern is also critical. If the duration is not $3 \mathrm{~ms}$ but $<2 \mathrm{~m}$ or $>4 \mathrm{~ms}$ malignant cells respond as if they were sham field-exposed. The physical chemical bases for this temporal particularity could be related to the two step energy boundaries associated with calcium channels or with the unusual features of the proton channel.

It is relevant that the digitized quantitative electroencephalogram of the right hemisphere (but not the left) of a person with exceptional and qualitatively different memory and information access abilities displayed spectral power densities profiles that are very similar to the Thomas pattern. In fact when this Harribance Configuration was applied as magnetic fields through geometry of solenoids similar to the present experiment [22] for $1 \mathrm{hr}$ per day for 5 days to melanoma cells they exhibited a comparable suppression effect. Applications of the left hemispheric fields from this person's brain produced effects that did not differ significantly from sham-field exposures. These results indicate that some human brains may exhibit electromagnetic configurations that when applied within the appropriate geometry can suppress the growth of malignant cells as effectively as theoretically generated patterns. Interestingly, 3 ms point durations are equivalent to about $330 \mathrm{~Hz}$ which is the upper boundary for the fast frequencies measured in human and rat epileptic brain [23].

If one assumes that the inhibitory effect upon the processes that contribute to aberrant proliferation in cancer cells is similar to the temporal structure intrinsic to the Thomas pattern that shares some feature with the Harribance Configuration then application of the LTP pattern to the latter should also produce cancellation. Search for previous research with Mr. Harribance indicated he had been exposed transcerebrally (across the temporal lobes) to several different magnetic patterns. The configuration that evoked the greatest change in his subjective experiences had been the LTP pattern [24].

Appropriately patterned magnetic fields, such as the LTP configuration, are not the only stimuli that can cancel the suppression effects of the Thomas pattern. Murugan et al. [25] exposed melanoma cells as well as sectioned planarian to either LED (light emitting diode) generated red, blue, or green light pulsed as a Thomas pattern ( $3 \mathrm{~ms}$ duration), the Thomas pattern as a magnetic field only, or the combination of the two. Whereas the Thomas pattern only produced the typical suppression of melanoma cell growth (but enhanced regeneration of planarian) and blue simulated this condition green light elicited minimal effects. When the green light was pulsed with the magnetic field as the Thomas pattern the growth suppression effects were completely eliminated in a manner similar to the LTP+Thomas influence noted in the present experiments.

Isolating the different classes of stimuli that can eliminate the beneficial effects of applied magnetic fields to malignant cells will be required to ensure the maximum efficiency. In addition identification of these stimuli and the mechanisms, through which the growth inhibitory effects are blocked, may reveal not only the basic mechanisms involved with malignancy but the physical chemical principles upon which they are based. It may also reveal a recondite relationship between the development "switch" for biomolecular cascades that trigger LTP induction and malignant potential [26] as well as the functional transition between epileptiform-like activity and associated memory in the hippocampus [27]. We suggest this may be relevant for an alternative explanation for extraordinarily high correlation between late onset complex partial (temporal lobe) epilepsy and the presence of tumors in human beings.

\section{Acknowledgements}

We thank Dr. W. E. Bosarge, CEO of Capital Industries, Inc and the Bosarge Family Organization for their support of this research.

\section{References}

Karbowski LM, Murugan NJ, Lafrenie RM, Persinger MA (2016) Experimental demonstration that Aharanov-Bohm phase shifts voltages in optical coupler circuits of tuned patterned magnetic fields are critical for inhibition of malignant cell growth. Journal of Advances in Physics 11: 3557-3563.

2. Costa FP, de Oliveria AC, Meirelles R, Machado MCC, Zanesco Z, et al. (2011) Treatment of advanced hepatocellular carcinoma with very low levels of amplitude-modulated electromagnetic fields. British Journal of Cancer 105: 640-648.

3. Yamaguschi S, Ogiue-Ikeda M, Sekino M, Ueno S (2005) Effects of pulsed magnetic stimulation on tumor development and immune functions in mice. Bioelectromagnetics 27: 64-72.

4. Nie Y, Du L, Mou Y, Weng L, Du Y, et al. (2013) Effect of low frequency magnetic fields on melanoma: tumor inhibition and immune modulation. BMC Cancer 13: 582.

5. Rose GM, Dunwiddie TV (1986) Induction of hippocampal longterm potentiation using physiologically patterned stimulation. Neuroscience Letters 69: 244-248.

6. Whitlock JR, Heynen AJ, Shuler MG, Bear MF (2006) Learning induces long-term potentiation in the hippocampus. Science 313: 1093-1097.

7. Persinger MA, Lafrenie RM (2014) The cancer cell plasma membrane potentials as energetic equivalents to astrophysical properties. International Letters of Chemistry, Physics and Astronomy 17: 67-77.

8. Persinger MA (2015) Thixtotropic phenomena in water: quantitative indicators of Casimir-magnetic transformations from vacuum oscillations (virtual particles). Entropy 17: 6200-6212.

9. Buckner CA (2011) Effects of electromagnetic fields on biologic processes are spatial and temporal dependent. Ph.D. Thesis, Laurentian University.

10. Koren SA, Bosarge WE, Persinger MA (2015) Magnetic fields generated by optical coupler circuits may also be containment loci for entanglement of P-N junction-plasma cell membrane photons within exposed living systems. International Letters of Chemistry, Physics and Astronomy 3: 84-105. 
11. Martin LJ, Koren SA, Persinger MA (2004) Thermal analgesic effects from weak, complex magnetic fields and pharmacological interactions. Pharmacology, Biochemistry and Behavior 78: 217-227.

12. Persinger MA, Koren SA (2007) A theory of neurophysics and quantum neuroscience: implications for brain function and the limits of consciousness. International Journal of Neuroscience 117: $157-175$

13. Koren SA, Dotta BT, Persinger MA (2014) Experimental photon doubling as a possible local inference of the Hubble parameter. The Open Astronomy Journal 7: 1-6.

14. Buckner CA, Buckner AL, Koren SA, Persinger MA, Lafrenie RM (2015) Inhibition of cancer cell growth by exposure to a specific time-varying electromagnetic field involves T-type calcium channesl. PLOS One.

15. Su H, Sochivko D, Becker A, Chen J, Jiang Y, et al. (2002) Upregulation of T-type $\mathrm{Ca}+2$ channel causes long-lasting modification of neuronal firing mode after status epilepticus. Journal of Neuroscience 22: 3645-3655.

16. Wolfart J, Roeper J (2002) Selective coupling of T-type calcium channels to SK potassium channels prevents intrinsic burst firing in dopaminergic midbrain neurons. The Journal of Neuroscience 22: 3404-3413.

17. Bozzi Y, Dunleavy M, Henshall D (2011) Cell signaling underlying epileptic behavior. Frontiers in Behavioral Neuroscience.

18. St-Pierre LS, Persinger MA (2003) Conspicuous histomorphological anomalies in the hippocampal formation of rats exposed prenatally to a complex sequenced magnetic field within the nanoTesla range. Perceptual and Motor Skills 97: 1307-1314.

19. Mach QH, Persinger MA (2009) Behavioral changes with brief exposures to weak magnetic fields patterned to stimulate longterm potentiation. Brain Research 1261: 45-53.
20. Karbowski LM, Murugan NJ, Koren SA, Persinger MA (2015) Seeking the source of transience for a unique magnetic field pattern that completely dissolves cancer cells in vitro. Journal of Biomedical Science and Engineering 8: 531-543.

21. Karbowski LM, Persinger MA (2015) Variable viscosity of water as the controlling factor in energetic quantities that control living systems: physicochemical and astronomical interactions. International Letters of Chemistry, Physics and Astronomy 4: 1-9.

22. Karbowski LM, Harribance SL, Buckner CA, Mulligan BP, Koren SA, et al. (2012) Digitized quantitative electroencephalographic patterns applied as magnetic fields inhibit melanoma cell proliferation in culture. Neuroscience Letters 523: 131-134.

23. Staba RJ, Wilson CL, Bragin A, Fried I, Engel J (2002) Quantitative analysis of high-frequency oscillations $(80$ to $500 \mathrm{~Hz}$ ) recorded in human epileptic hippocampus and entorhinal cortex. Journal of Neurophysiology 88: 1743-1752.

24. Cook CM, Persinger MA (1997) Experimental induction of the "sensed presence" in normal subjects and an exceptional subject. Perceptual and Motor Skills, 75: 683-693.

25. Murugan NJ, Karbowski LM, Persinger MA (2016) Synergistic interactions between temporal coupling of complex light and magnetic pulses upon melanoma cell proliferation and planarian regeneration. Electromagnetic Biology and Medicine, in press.

26. Yasuda H, Barth AL, Stellwagen D, Malenka RC (2003) A developmental switch in the signaling cascades for LTP induction. Nature neuroscience 6: 15-17.

27. Wallenstein GV, Hasselmo ME (1997) Functional transitions between epileptiform-like activity and associative memory in hippocampal region CA3. Brain Research Bulletin 43: 485-493. 